\title{
On isomorphisms and invariants of finite dimensional complex filiform Leibniz algebras
}

\begin{abstract}
In this article, we propose an approach classifying a class of filiform Leibniz algebras. The approach is based on algebraic invariants. The method allows to classify all filiform Leibniz algebras (including filiform Lie algebras) in a given fixed dimensional case.
\end{abstract}

Keyword: Isomorphism; Filiform Leibniz algebra; Isomorphism criterion; Lie algebra; Natural gradation 